\title{
THE EXTINCTION TIME OF A SUBCRITICAL BRANCHING PROCESS RELATED TO THE SIR EPIDEMIC ON A RANDOM GRAPH
}

\author{
PETER WINDRIDGE, ${ }^{*}$ Queen Mary University of London
}

\begin{abstract}
We give an exponential tail approximation for the extinction time of a subcritical multitype branching process arising from the SIR epidemic model on a random graph with given degrees, where the type corresponds to the vertex degree. As a corollary we obtain a Gumbel limit law for the extinction time, when beginning with a large population. Our contribution is to allow countably many types (this corresponds to unbounded degrees in the random graph epidemic model, as the number of vertices tends to $\infty$ ). We only require a second moment for the offspring-type distribution featuring in our model.
\end{abstract}

Keywords: Multitype branching process; exponential tail approximation; Gumbel; SIR epidemic

2010 Mathematics Subject Classification: Primary 60J80; 92D30

Secondary $05 \mathrm{C} 80 ; 60 \mathrm{~J} 28$

\section{Introduction}

In this paper we consider a continuous-time Markov multitype branching process $Z=$ $\left(Z_{t}(k) ; t \geq 0, k=1,2, \ldots\right)$ arising from the susceptible $\rightarrow$ infective $\rightarrow$ recovered (SIR) epidemic model on a random graph with given degrees. (We mention this connection only as motivation and do not explain it in detail. If desired, the reader can consult [2] or [7] for a construction in which the branching process studied here is apparent. See also Remark 2 below.)

Individuals in $Z$ are thought of as infective hosts carrying a number of spores. An individual's type $k \geq 1$ is simply the number of spores it has. (We ignore individuals with no spores.) Each spore, at a given rate $\beta>0$, is released and gives rise to a new infective individual. The new individual has a random type (i.e. number of spores), $J$ say, chosen according to some given probability distribution, denoted $\left(p_{j}\right)_{j=0}^{\infty}$. (We allow $p_{0}>0$, and $J=0$ means that no new infective is produced.) This leaves one individual of type $k-1$ (representing the original individual after losing a spore, assuming $k \geq 2$ ), and another with type $J$ (assuming $J \geq 1$ ). Furthermore, each individual (including its hosted spores) is removed from the population at rate $\rho \geq 0$, leaving nothing in its stead, regardless of type. Thus, an individual of type $k$ has an exponentially distributed lifetime of rate $\rho+\beta k$, and is replaced by 0,1 , or 2 individuals.

This is a standard form of multitype branching process. Classical theory provides an exponential tail approximation for the extinction time in the subcritical case, at least when there are finitely many types, i.e. $\left(p_{j}\right)_{j=0}^{\infty}$ has finite support (see [5], and [3, Chapter V, Theorem 11.1] for the single-type result of Sewastjanow). In the general denumerable case, extinction itself is more delicate, for example extinction can be almost sure even though the expected population

Received 23 September 2014; revision received 30 November 2014.

* Current address: HSBC, 8 Canada Square, London, E14 5HQ, UK. Email address: pete@ windridge.org.uk 
size tends to $\infty$ [4]. Tail approximations have been made in special cases, such as birth distributions of linear-fractional form [9]. However, there does not seem to be an applicable result for our rather simple model. In this paper we require only that $\left(p_{j}\right)_{j=0}^{\infty}$ has a second moment.

Denote by

$$
q_{k}(t)=\mathbb{P}\left(Z_{t} \neq 0 \mid Z_{0}(i)=\delta_{k i}, i \geq 1\right),
$$

the probability that the process survives till time $t \geq 0$ when it begins with a single host carrying $k \geq 1$ spores. (We find it more intuitive to speak of hosts and their spore counts in the sequel, rather than using the branching process terminology of individuals and their type.) The exponential approximation result we prove is as follows.

Theorem 1. Suppose that $\mu:=\sum_{k=0}^{\infty} k p_{k}>0$,

$$
\lambda:=\rho+\beta(1-\mu)>0,
$$

and

$$
\sum_{k=0}^{\infty} k^{2} p_{k}<\infty
$$

Then there exists a constant $\hat{c} \in(0,1]$ such that, for any $a<\min \{\lambda, \beta\}$,

$$
q_{k}(t)=\hat{c} k \mathrm{e}^{-\lambda t}\left(1+O\left(k \mathrm{e}^{-a t}\right)\right) \text { as } t \rightarrow \infty
$$

for all $k \geq 1$.

The condition in (1) means that $Z$ is subcritical. In the omitted case of $\mu=0$, no new hosts occur and $q_{k}(t)=\mathrm{e}^{-\rho t}\left(1-\left(1-\mathrm{e}^{-\beta t}\right)^{k}\right)$ is just the probability that the initial host is still present and at least one of its spores remains.

Remark 1. Any value of $\hat{c} \in(0,1]$ is possible. Indeed, if $\rho=0$ then all spores behave independently and form a single-type branching process. The $p_{0}+p_{1}+p_{2}=1$ case is linear fractional [1, Section III.5, p. 109] and $q_{1}(t)$ can be computed explicitly. More specifically, $p_{0}+p_{2}=1$ corresponds to a linear birth and death chain and for $p_{0} \neq p_{2}$, we have

$$
q_{1}(t)=\frac{\left(p_{0}-p_{2}\right) \mathrm{e}^{-\beta\left(p_{0}-p_{2}\right) t}}{p_{0}-p_{2} \mathrm{e}^{-\beta\left(p_{0}-p_{2}\right) t}} .
$$

Thus, the leading constant in Theorem 1 is $\hat{c}=1-p_{2} / p_{0}$ for any $p_{2}<p_{0}$.

Theorem 1 can be used to examine the distribution of the duration of a subcritical epidemic where there are initially large number of infective hosts. The utility of such a result is perhaps not immediately apparent, so we remark that the situation described also arises at the end of a major epidemic, when an outbreak has become so large that it starts shrinking due to there being few remaining susceptible individuals.

Corollary 1. Adopt the setting of Theorem 1. Suppose that for each $n \geq 1$, we have a sequence $z_{k}, k \geq 1$ of natural numbers such that $\sum_{k=0}^{\infty} k z_{k} \rightarrow \infty$, and

$$
\sum_{k=0}^{\infty} k^{2} z_{k}=o\left(\sum_{k=0}^{\infty} k z_{k}\right)^{1+(a / \lambda)} \text { as } n \rightarrow \infty
$$

for some a $>0$ satisfying the condition in Theorem 1. 
Suppose that $Z_{0}(k)=z_{k}$ for every $k$ and $T:=\inf \left\{t \geq 0: Z_{t}=0\right\}$. Then, for any fixed $w \in \mathbb{R}$,

$$
\mathbb{P}\left(\lambda T \leq \ln \left(\hat{c} \sum_{k=0}^{\infty} k z_{k}\right)+w\right) \rightarrow \mathrm{e}^{-\mathrm{e}^{-w}} \text { as } n \rightarrow \infty .
$$

The double exponential distribution on the right-hand side of (5) is known as the Gumbel distribution. It arises from taking the maximum of a large number of independent random variables with exponential tails, and thus is common in the context of branching process extinction times [5], [6], [8].

Remark 2. When studying the SIR epidemic on a random graph with given degree sequence, one typically constructs (or 'reveals') relevant parts of the graph while the disease spreads, via a device known as the configuration model; see [7] and the references therein. For the benefit of readers familiar with the configuration model approach, it is worth noting that the 'spores' in this paper correspond to half-edges, and a 'host' is just a vertex in the graph. To apply our result, the probability distribution $\left(p_{k}\right)_{k=0}^{\infty}$ should be a size-biased transform of the graph degree distribution. In particular, our second moment condition translates to a third moment requirement for the vertex degree distribution. The details of a pathwise coupling, and conditions needed for it to hold with high probability, are left for future work.

The proofs of Theorem 1 and Corollary 1, in Sections 2 and 3, respectively, occupy the remainder of this paper.

\section{Proof of Theorem 1}

The general idea is to show that $q_{k}(t) \sim k q_{1}(t)$, as $t \rightarrow \infty$, by controlling the dependencies between spores of the same host; see Lemma 1 below. First we need preliminary bounds on $q_{1}(t)$. Let us fix $a<\min \{\lambda, \beta\}$. Suppose that initially there is a single host with one spore. Let $R$ denote its $\operatorname{Exp}(\rho)$ removal time, and $F$ the $\operatorname{Exp}(\beta)$ release time of its single spore. The process survives till a given time $t>0$ if and only if either the spore is released before $t$ (and necessarily before the host is removed) and its progeny persist till time $t$, or neither the spore is released nor the host removed by $t$. Thus,

$$
\begin{aligned}
q_{1}(t) & =\int_{0}^{t} \sum_{k=0}^{\infty} p_{k} q_{k}(t-f) \mathbb{P}(F \in \mathrm{d} f) \mathbb{P}(R>f)+\mathbb{P}(F, R>t) \\
& =\int_{0}^{t} \sum_{k=0}^{\infty} p_{k} q_{k}(t-f) \beta \mathrm{e}^{-(\rho+\beta) f} \mathrm{~d} f+\mathrm{e}^{-(\rho+\beta) t},
\end{aligned}
$$

from which we obtain the differential equation

$$
q_{1}^{\prime}(t)=-(\rho+\beta) q_{1}(t)+\beta \sum_{k=0}^{\infty} p_{k} q_{k}(t), \quad t \geq 0, q_{1}(0)=1
$$

This also follows from the Kolmogorov backwards equations, but deriving it from the integral is a useful warmup for the calculations below.

Now suppose there is initially a host with $k \geq 2$ spores. Survival of the process till time $t$ implies that at least one of the $k$ initial spores, or its progeny, persist till $t$. It follows that 
$q_{k}(t) \leq k q_{1}(t)$. Using this inequality with (6) yields

$$
q_{1}^{\prime}(t) \leq-(\rho+\beta) q_{1}(t)+\beta \sum_{k=0}^{\infty} k p_{k} q_{1}(t)=-\lambda q_{1}(t),
$$

and so $\mathrm{e}^{\lambda t} q_{1}(t)$ is nonincreasing in $t$. It is positive and so the limit

$$
\hat{c}:=\lim _{t \rightarrow \infty} \mathrm{e}^{\lambda t} q_{1}(t)
$$

exists. We have $\hat{c} \leq 1$ by monotonicity of $\mathrm{e}^{\lambda t} q_{1}(t)$ and the fact that $q_{1}(0)=1$.

It will later transpire that $\hat{c}>0$. For now we will lower bound $q_{1}(t)$ by truncating the spore count distribution $\left(p_{k}\right)_{k=0}^{\infty}$. More precisely, take any positive $\varepsilon<\min \{\lambda, \beta\}-a$, and choose $k_{0} \geq 1$ large enough that $\sum_{k=1}^{k_{0}} k p_{k}>\mu-\varepsilon / \beta$. Our branching process $Z$ dominates a modified process in which spore counts of new hosts are distributed as $J \mathbf{1}_{\left\{J \leq k_{0}\right\}}$, where $J \sim\left(p_{k}\right)_{k=0}^{\infty}$. This modified process has finitely many types, and so the exponential tail approximation of [5] applies, with decay rate $\rho+\beta-\beta \sum_{k=1}^{k_{0}} k p_{k}<\lambda+\varepsilon$. In particular, there exists $c_{1}=c_{1}(\varepsilon)>0$ such that

$$
q_{1}(t) \geq c_{1} \mathrm{e}^{-(\lambda+\varepsilon) t} \quad \text { for all } t \geq 0 .
$$

Lemma 1. For all $k \geq 1$, we have

$$
q_{k}(t)=k q_{1}(t)\left(1+O\left(k \mathrm{e}^{-a t}\right)\right) \text { as } t \rightarrow \infty \text {. }
$$

Proof. As already mentioned we have $q_{k}(t) \leq k q_{1}(t)$. For the lower bound, suppose that there is initially a single host with $k \geq 2$ spores. Let $T_{i}, i=1, \ldots, k$ denote the total time that spore $i$, or its progeny, persist for. Thus,

$$
q_{k}(t)=\mathbb{P}\left(\bigcup_{i=1}^{k}\left\{T_{i}>t\right\}\right)
$$

The Bonferroni inequality yields

$$
q_{k}(t) \geq k q_{1}(t)-k^{2} \mathbb{P}\left(T_{1}, T_{2}>t\right),
$$

where we used $\mathbb{P}\left(T_{1}>t\right)=q_{1}(t)$, and the fact that any pair of times have the same joint distribution. If $\rho=0$ then $T_{1}$ and $T_{2}$ are independent and there is nothing to prove. In the sequel, we assume that $\rho>0$ and control the dependency between the $T_{i}$ using the fact that the progeny of different spores behave independently.

Suppose that $F_{1}$ and $F_{2}$ denote the independent $\operatorname{Exp}(\beta)$ release times of spores 1 and 2, and $R$ denotes the $\operatorname{Exp}(\rho)$ removal time of the host. Then, ignoring the null events $\{R<t$ and $\left.T_{i}, F_{i}>t\right\}$, we have

$$
\begin{aligned}
\mathbb{P}\left(T_{1}, T_{2}>t\right)= & \mathbb{P}\left(R, F_{1}, F_{2}>t\right)+2 \mathbb{P}\left(T_{1}, F_{2}, R>t, \text { and } F_{1}<t\right) \\
& +\mathbb{P}\left(T_{1}, T_{2}>t, \text { and } F_{1}, F_{2}<t\right) .
\end{aligned}
$$

The first probability on the right-hand side is simply $\mathrm{e}^{-(\rho+2 \beta) t}$. The second probability can be bounded by writing it as an integral against the spore release time, and then using $q_{k}(t) \leq k q_{1}(t)$, 
$\mu=\sum_{k=0}^{\infty} k p_{k}$, and $q_{1}(t) \leq \mathrm{e}^{-\lambda t}$ as follows:

$$
\begin{aligned}
\mathbb{P}\left(T_{1}, F_{2}, R>t, \text { and } F_{1}<t\right) & =\mathrm{e}^{-(\rho+\beta) t} \int_{0}^{t} \sum_{k=0}^{\infty} p_{k} q_{k}(t-f) \mathbb{P}\left(F_{1} \in \mathrm{d} f\right) \\
& \leq \mu \mathrm{e}^{-(\rho+\beta) t} \int_{0}^{t} q_{1}(t-f) \beta \mathrm{e}^{-\beta f} \mathrm{~d} f \\
& \leq \mu \beta \mathrm{e}^{-(\rho+\beta+\lambda) t} \int_{0}^{t} \mathrm{e}^{(\lambda-\beta) f} \mathrm{~d} f \\
& =\mu \beta \mathrm{e}^{-(\rho+\beta+\lambda) t}\left(\frac{\mathrm{e}^{(\lambda-\beta) t}-1}{\lambda-\beta}\right)
\end{aligned}
$$

assuming $\lambda \neq \beta$, otherwise the last integral evaluates to $t$. In both cases,

$$
\begin{aligned}
\mathbb{P}\left(T_{1}, F_{2}, R>t, \text { and } F_{1}<t\right) & =O\left(\mathrm{e}^{-(\rho+2 \beta) t}+(1+t) \mathrm{e}^{-(\rho+\beta+\lambda) t}\right) \\
& =O\left(\mathrm{e}^{-(\rho+2 \beta) t}+\mathrm{e}^{-(\beta+\lambda) t}\right) .
\end{aligned}
$$

Finally, we turn to the third probability on the right-hand side of (10). Consider the probability $g(t, r)$ that a given spore is released before time $r>0$ (assuming the host is not removed first) and has progeny who persist till time $t \geq r$. Repeating calculations similar to those in (11) shows it satisfies

$$
g(t, r)=\int_{0}^{r} \sum_{k=0}^{\infty} p_{k} q_{k}(t-f) \mathbb{P}\left(F_{1} \in \mathrm{d} f\right) \leq \mu \beta \mathrm{e}^{-\lambda t}\left(\frac{\mathrm{e}^{(\lambda-\beta) r}-1}{\lambda-\beta}\right) \text { for } \lambda \neq \beta,
$$

otherwise the bracketted term is $r$. Thus, the probability that two given spores are released before time $r$ (ignoring removal of the host) and bear progeny persisting till time $t \geq r$ satisfies

$$
g(t, r)^{2} \leq c_{2} \mathrm{e}^{-2 \lambda t}\left(\mathrm{e}^{2(\lambda-\beta) r}+1+r^{2}\right)
$$

for some constant $c_{2}>0$. Integrating (12) against the removal time density yields

$$
\begin{aligned}
\mathbb{P}\left(T_{1}, T_{2}>t, \text { and } F_{1}, F_{2}<t\right) & =\int_{0}^{t} g(t, r)^{2} \mathbb{P}(R \in \mathrm{d} r)+g(t, t)^{2} \mathbb{P}(R>t) \\
& =O\left(\mathrm{e}^{-2 \lambda t}+\mathrm{e}^{-(\rho+2 \beta) t}+\left(1+t^{2}\right) \mathrm{e}^{-(2 \lambda+\rho) t}\right) \\
& =O\left(\mathrm{e}^{-2 \lambda t}+\mathrm{e}^{-(\rho+2 \beta) t}\right) .
\end{aligned}
$$

Armed with these estimates, we return to (10) and find that

$$
\mathbb{P}\left(T_{1}, T_{2}>t\right)=O\left(\mathrm{e}^{-(\rho+2 \beta) t}+\mathrm{e}^{-(\beta+\lambda) t}+\mathrm{e}^{-2 \lambda t}\right),
$$

and using (7), we have

$$
\frac{\mathbb{P}\left(T_{1}, T_{2}>t\right)}{q_{1}(t)}=O\left(\mathrm{e}^{-(\rho+2 \beta-\lambda-\varepsilon) t}+\mathrm{e}^{-(\beta-\varepsilon) t}+\mathrm{e}^{-(\lambda-\varepsilon) t}\right) .
$$

Now, $\rho+2 \beta-\lambda=\beta(1+\mu)>\beta$. So, each negative exponent in (13) is at least $a$ by our choice of $\varepsilon$. The desired relationship (8) now follows from the Bonferroni inequality (9). 
We will now prove that $\hat{c}>0$, i.e. that $q_{1}(t)$ really does decay like $\mathrm{e}^{-\lambda t}$. Apply Lemma 1 to obtain

$$
\begin{aligned}
\sum_{k=0}^{\infty} p_{k} q_{k}(t) & \geq \sum_{k=0}^{\infty} k p_{k} q_{1}(t)-O\left(q_{1}(t) \mathrm{e}^{-a t} \sum_{k=0}^{\infty} k^{2} p_{k}\right) \\
& =q_{1}(t)\left(\mu+O\left(\mathrm{e}^{-a t}\right)\right),
\end{aligned}
$$

using the assumption (2) that $\sum_{k=0}^{\infty} k^{2} p_{k}<\infty$. Combining this with (6) for $q_{1}(t)$ yields

$$
\begin{aligned}
\frac{\mathrm{d}}{\mathrm{d} t}\left(\ln q_{1}(t)+\lambda t\right) & =\frac{q_{1}^{\prime}(t)}{q_{1}(t)}+\lambda \\
& =-(\rho+\beta)+\frac{\beta}{q_{1}(t)} \sum_{k=0}^{\infty} p_{k} q_{k}(t)+\lambda \\
& \geq-c_{3} \mathrm{e}^{-a t}
\end{aligned}
$$

for some constant $c_{3}>0$. Integrating both sides, we obtain

$$
\ln q_{1}(t)+\lambda t \geq-c_{3} \int_{0}^{t} \mathrm{e}^{-a s} \mathrm{~d} s \geq-\frac{c_{3}}{a} .
$$

Hence, $\hat{c}=\lim _{t \rightarrow \infty} \mathrm{e}^{\lambda t} q_{1}(t) \geq \exp \left(-c_{3} / a\right)>0$.

Finally, $\mathrm{e}^{\lambda t} q_{1}(t)$ is nonincreasing so $q_{1}(t) \geq \hat{c} \mathrm{e}^{-\lambda t}$. Moreover, using (14) again, we obtain

$$
\ln \left(\mathrm{e}^{\lambda t} q_{1}(t)\right) \leq c_{3} \int_{t}^{\infty} \mathrm{e}^{-a s} \mathrm{~d} s+\lim _{s \rightarrow \infty} \ln \left(\mathrm{e}^{\lambda s} q_{1}(s)\right)=O\left(\mathrm{e}^{-a t}\right)+\ln \hat{c} .
$$

But $\exp \left(O\left(\mathrm{e}^{-a t}\right)\right)=1+O\left(\mathrm{e}^{-a t}\right)$ as $t \rightarrow \infty$ and so (3) holds for $k=1$. The result for $k \geq 2$ follows from (8).

Remark 3. The proof in [5] (for finitely many types) uses a linear approximation to (6) its analogues for $q_{k}, k \geq 2$. A general result about perturbations of linear equations is then applied to derive (3). We took a different approach here due to not finding a suitable perturbation result for infinite systems of differential equations.

\section{Proof of Corollary 1}

Take $t=t(n):=\lambda^{-1}\left(\ln \left(\hat{c} \sum_{k=0}^{\infty} k z_{k}\right)+w\right)$, so $t>0$ for large enough $n$. Using the branching property, Theorem 1, and finally assumption (4), we have

$$
\begin{aligned}
\ln \mathbb{P}(T \leq t) & =\ln \prod_{k=0}^{\infty}\left(1-q_{k}(t)\right)^{z_{k}} \\
& =\sum_{k=0}^{\infty} z_{k} \ln \left(1-q_{k}(t)\right) \\
& \leq-\sum_{k=0}^{\infty} z_{k} q_{k}(t)
\end{aligned}
$$




$$
\begin{aligned}
& \leq-\hat{c} \mathrm{e}^{-\lambda t} \sum_{k=0}^{\infty} k z_{k}+O\left(\mathrm{e}^{-(a+\lambda) t} \sum_{k=0}^{\infty} k^{2} z_{k}\right) \\
& =-\mathrm{e}^{-w}+O\left(\frac{\sum_{k=0}^{\infty} k^{2} z_{k}}{\left(\sum_{k=0}^{\infty} k z_{k}\right)^{1+(a / \lambda)}}\right) \\
& \rightarrow-\mathrm{e}^{-w} \text { as } n \rightarrow \infty .
\end{aligned}
$$

On the other hand, (4) implies that the maximum number of spores $z_{*}:=\max \left\{k: z_{k} \geq 1\right\}$ of any initial host satisfies $z_{*}=o\left(\sum_{k=0}^{\infty} k z_{k}\right)$. So, for any $k \leq z_{*}$, we have

$$
q_{k}(t) \leq k q_{1}(t) \leq k \mathrm{e}^{-\lambda t} \leq \frac{z_{*}}{\mathrm{e}^{w} \hat{c} \sum_{k=0}^{\infty} k z_{k}} \rightarrow 0 \quad \text { as } n \rightarrow \infty
$$

This implies that $q_{k}(t)<\frac{1}{2}$ eventually (for any $k$ with $z_{k} \geq 1$ ) and, consequently,

$$
\ln \left(1-q_{k}(t)\right) \geq-q_{k}(t)\left(1+q_{k}(t)\right) \geq-q_{k}(t)-k^{2} \mathrm{e}^{-2 \lambda t},
$$

using the inequality $\ln (1-h) \geq-h(1+h)$ for $h \leq \frac{1}{2}$. It follows that

$$
\ln \mathbb{P}(T \leq t)=\sum_{k=0}^{\infty} z_{k} \ln \left(1-q_{k}(t)\right) \geq-\sum_{k=0}^{\infty} z_{k} q_{k}(t)-\frac{\sum_{k=0}^{\infty} k^{2} z_{k}}{\left(\mathrm{e}^{w} \hat{c} \sum_{k=0}^{\infty} k z_{k}\right)^{2}} \rightarrow-\mathrm{e}^{-w},
$$

as $n \rightarrow \infty$, as in the upper bound.

\section{Acknowledgements}

Funded by EPSRC (grant no. EP/J004022/2. Principal investigator Malwina Luczak). The author thanks Svante Janson and Malwina Luczak for useful comments.

\section{References}

[1] Athreya, K. B. And Ney, P. E. (2004). Branching Processes. Dover, Mineola, NY.

[2] Bohman, T. ANd Picollelli, M. (2012). SIR epidemics on random graphs with a fixed degree sequence. Random Structures Algorithms 41, 179-214.

[3] Harris, T. E. (1963). The Theory of Branching Processes. Springer, Berlin.

[4] Hautphenne, S., Latouche, G. and Nguyen, G. (2013). Extinction probabilities of branching processes with countably infinitely many types. Adv. Appl. Prob. 45, 1068-1082.

[5] Heinzmann, D. (2009). Extinction times in multitype Markov branching processes. J. Appl. Prob. 46, $296-307$.

[6] Jagers, P., Klebaner, F. C. And Sagitov, S. (2007). On the path to extinction. Proc. Nat. Acad. Sci. USA 104, 6107-6111.

[7] JANSON, S., LuCZAK, M. AND WindRIDGe, P. (2014). Law of large numbers for the SIR epidemic on a random graph with given degrees. Random Structures Algorithms 45, 724-761.

[8] PAKes, A. G. (1989). Asymptotic results for the extinction time of Markov branching processes allowing emigration. I. Random walk decrements. Adv. Appl. Prob. 21, 243-269.

[9] Sagitov, S. (2013). Linear-fractional branching processes with countably many types. Stoch. Process. Appl. 123, 2940-2956. 